\title{
Optimal Settings for Narrow-Band Signal Measurements Used for Exposure Assessment Around GSM Base Stations
}

\author{
Christof Olivier and Luc Martens, Member, IEEE
}

\begin{abstract}
The use of frequency-selective measurements for the assessment of the worst-case exposure to electromagnetic fields radiated by GSM base stations is widely accepted to check the compliance with the existing regulations when multiple frequency sources are present. In this paper, the influence of different settings of the spectrum analyzer (resolution bandwidth, sweep rate, and detector) on the accuracy of power measurements has been studied with simulations by using a simple model for the spectrum analyzer and the global system for mobile communications (GSM) signal. The effect of adjacent frequency channels, time modulated signals, and down link power control have been investigated. Based on the obtained simulation results, optimal spectrum analyzer settings have been proposed for the exposure assessment around GSM base stations. As a final result, the accuracy of the proposed optimal settings for the spectrum analyzer has been determined.
\end{abstract}

Index Terms-Electromagnetic radiation, land mobile radio cellular systems, spectral analysis.

\section{INTRODUCTION}

$\mathbf{M}$ OBILE communications have experienced an enormous growth during the past decade. To deal with the raising number of mobile subscribers and the increasing demand for bandwidth, mobile operators have to install more and more base stations. More people are, thus, confronted with a base station in their neighborhood and are concerned about possible adverse health effects of the electromagnetic radiation emitted by the base stations. To deal with these concerns and to protect the population against excessive exposure to electromagnetic fields (EMF), authorities have issued regulations for the implantation and exploitation of base stations for mobile communications. These regulations should guarantee that the exposure to EMF never exceeds the issued limits (e.g., [1]), which are based on recommendations by international expert organizations [2]-[4]. In order to check the compliance of cellular base stations with these limits, several procedures have been proposed [5]-[7] and are now being standardized. In these procedures, it is recommended that narrow-band measurements (e.g., with a spectrum analyzer) are used when multiple frequency sources are present on a location. This enables to characterize the contribution of each emitting source to the total exposure and to determine the

Manuscript received March 31, 2003; revised March 31, 2004.

C. Olivier is with the Department of Information Technology, Ghent University, B-9000 Ghent, Belgium, and with the Fund for Scientific Research Flanders (F.W.O.-Vlaanderen), Belgium.

L. Martens is with the Department of Information Technology, Ghent University, B-9000 Ghent, Belgium.

Digital Object Identifier 10.1109/TIM.2004.838114 responsible for an elevated exposure level. Since most of the normative documents prescribe that the exposure to EMF has always to comply to the limits, it is necessary that the worst-case exposure is estimated from the measurements. The measurement results should, thus, provide for each source of EMF the maximum exposure that it could ever produce on a certain location. Since, for each source of EMF, a distinct frequency band has been allocated, the maximum possible power in a certain frequency band should be determined.

In this paper, some issues regarding narrow-band measurements of digital modulated communication signals are discussed, within the context of compliance verification of base stations to the exposure limits. The focus of the study will be on the global system for mobile communications (GSM) signal, since this is now the most widespread mobile telephony system. However, the used methods, the obtained results, and the underlaying principles could be extended to other mobile communication systems.

In this paper, the behavior of the measured power level of a GSM signal as a function of the settings of the spectrum analyzer is explained, taking into account the characteristics of the GSM signal. To study the behavior, simple simulation models for the spectrum analyzer as well as for the GSM signal generator are used, enabling to characterize the influence of individual parameters and configurations on the measured power level. The main advantage of simulations compared to measurements is the ability to study individual parameters by keeping all other variables constant and, thus, characterizing the influence of the considered parameter. Simulations also permit the observation of signals and variables that are only visible inside the model, and thus, to determine the underlying reasons for a certain behavior. Moreover, simulations indicate theoretical bounds on the achievable accuracy, since imperfections of the spectrum analyzer (e.g., noise) can be neglected in the model. However, it still remains necessary to validate the results obtained from simulations by comparing them to measurements, although deviations can occur due to the simplifications made in the simulation models. Based on the observed properties of the GSM signal when measured with a spectrum analyzer, the optimal settings for the spectrum analyzer will be proposed. The spectral measurements should be able to accurately estimate within a reasonable time the maximum power that can be present in a GSM channel, taking into account that the transmitted power may change during the measurement period due to varying traffic and power control, and on the other hand, the measurement should be able to make distinction between adjacent channels. 


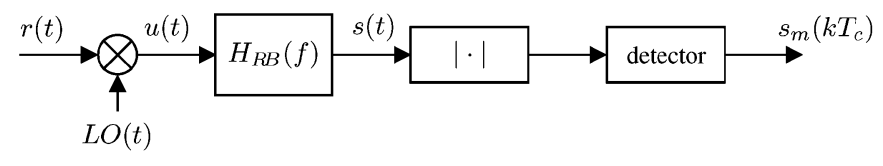

Fig. 1. Baseband model for the spectrum analyzer.

In Section II, the simulation models that have been used for the spectrum analyzer and for the GSM model are presented. The study of the different settings of the spectrum analyzer is given in Section III. In Section IV, the optimal settings for the spectrum analyzer to assess the exposure from a GSM base station are derived based on the simulation results.

\section{Simulation Models}

To be able to execute simulations at a relatively low sampling rate, the baseband representations of the passband signals are used, both for the spectrum analyzer as well as for the communication signal (i.e., the GSM signal).

\section{A. Spectrum Analyzer Model}

The baseband model for the spectrum analyzer that has been used is illustrated in Fig. 1. In the passband representation, the signal to be measured $r_{\mathrm{PB}}(t)$ is multiplied with the local oscillator signal $\mathrm{LO}_{\mathrm{PB}}(t)$ which is given by

$$
\mathrm{LO}_{\mathrm{PB}}(t)=\cos \left(2 \pi\left(f_{C}+f_{\mathrm{IF}}+\frac{\Delta f_{\mathrm{sp}}}{2 \Delta T_{\mathrm{sw}}} t\right) t\right)
$$

where $f_{C}$ is the center frequency of the spectrum analyzer, $f_{\mathrm{IF}}$ the intermediate frequency at which the resolution filter operates, $\Delta f_{\mathrm{sp}}$ the span of the spectrum analyzer, and $\Delta T_{\mathrm{sw}}$ the sweep time. The sweep rate $v_{\mathrm{sw}}$ is defined as $v_{\mathrm{sw}}=\Delta f_{\mathrm{sp}} / \Delta T_{\mathrm{sw}}$. The baseband representation of the sweeping signal of the local oscillator is

$$
\mathrm{LO}(t)=\frac{1}{2} \exp \left(-j 2 \pi \frac{\Delta f_{\mathrm{sp}}}{2 \Delta T_{\mathrm{sw}}} t^{2}\right)
$$

The mixed signal is then sent to the resolution filter with its representation in the frequency domain $H_{\mathrm{RF}}(f)$ and in the time domain $h_{\mathrm{RF}}(t)$

$$
\begin{aligned}
H_{\mathrm{RF}}(f) & =\exp \left(-\frac{f^{2}}{2 \sigma_{f}^{2}}\right) \\
h_{\mathrm{RF}}(t) & =\exp \left(-2 \pi^{2} \sigma_{f}^{2} t^{2}\right)
\end{aligned}
$$

where $\sigma_{f}$ is proportional to the resolution bandwidth (RBW)

$$
\sigma_{f}=\mathrm{RBW} / \sqrt{4 \ln 2} .
$$

Since the resolution filter has a certain time response, the sweep rate has an upper limit to enable the resolution filter to reach steady state

$$
v_{\mathrm{sw}}=\frac{\Delta f_{\mathrm{sp}}}{\Delta T_{\mathrm{sw}}}<\frac{\mathrm{RBW}^{2}}{c}
$$

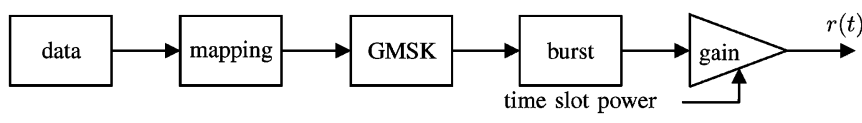

Fig. 2. Baseband model for the GSM signal.

where $c$ is a constant depending on the type of the spectrum analyzer. For an analog resolution filter, it is typically 2.5 [8]. The filtered signal $s(t)$ is then sent to the envelope detector, which is represented in the base band by the absolute value operator. The baseband representation of the signal leaving the envelope detector, is thus given by

$$
|s(t)|=\left|\frac{1}{2} \int_{-\infty}^{+\infty} r(t+u) e^{-j 2 \pi \frac{\Delta f_{\mathrm{sp}}}{2 \Delta T_{\mathrm{sw}}}(t+u)^{2}} e^{-2 \pi^{2} \sigma_{f}^{2} u^{2}} d u\right| .
$$

Finally, the detector will produce after each sample period $T_{s}$ a measurement value. The $k$ th measurement value $s_{m}\left(k T_{s}\right)$ will depend on the signal $|s(t)|$ during the previous interval $](k-$ 1) $\left.T_{s}, k T_{s}\right]$ and on the detector mode (sample, root mean square (RMS) or positive peak detection)

$s_{m}\left(k T_{s}\right)= \begin{cases}\left|s\left(k T_{s}\right)\right| & \text { sample } \\ \max _{\left.t \in](k-1) T_{s}, k T_{s}\right]}|s(t)| & \text { pos. peak } \\ \sqrt{\frac{1}{T_{s}} \int_{(k-1) T_{s}}^{k T_{s}}|s(t)|^{2} d t} & \text { RMS }\end{cases}$

Because the measured value at sample time $k T_{s}$ corresponds, for the positive peak and RMS detector, to the frequency content during the previous interval $\left.](k-1) T_{s}, k T_{s}\right]$, this interval is sometimes referred to as the frequency bin, with its width $\Delta f_{\mathrm{bin}}=v_{\mathrm{sw}} T_{s}$.

\section{B. GSM Signal Model}

The GSM signal model was implemented according to the specifications given in the ETSI/3GPP standards [9]-[12] and is given in Fig. 2. The signal on the physical layer is modeled starting from the burst formatting phase. The data stream entering the burst formatting block is considered to be completely random and is represented by a Bernoulli source with equal probability for the zeros and ones at a bit rate of approximately $201 \mathrm{~kb} / \mathrm{s}$.

This random bit stream is mapped on the burst by adding the tailing bit sequences, the guard bits, and the training sequence [9], resulting in a stream with a bit rate of approximately $270.8 \mathrm{~kb} / \mathrm{s}$. Only normal and dummy bursts are considered here; no model has been developed for frequency correction bursts nor synchronization bursts which are also used in the down link.

This formatted burst is then sent to the differential Gaussian minimum shift keying (GMSK) modulator $(\mathrm{BT}=0.3$, modulation rate of $270(5 / 6) \mathrm{kb} / \mathrm{s}$ ) [11]. The Phase 2+ GSM signal (for EDGE) using 8 PSK has not been implemented. Since the GSM frequency channels are separated by $200 \mathrm{kHz}$, the channel width of a GSM frequency channel is considered to be $200 \mathrm{kHz}$.

The power ramping during a burst is modeled in the burst block according to the specifications in [12]. The gain block 
provides the option to define the transmitted power for each time slot, so that the behavior of the GSM signal can be simulated for a traffic channel where not all the slots are used, or where the power of the slots varies because down link power control is enabled at the base station side.

The models were implemented in Simulink. Depending on the resolution bandwidth chosen, the sample rate of the simulations has been set between $50 \mathrm{~ns}$ for a resolution filter of $1 \mathrm{MHz}$ and $0.5 \mu$ s for a resolution filter of $1 \mathrm{kHz}$.

\section{Simulation Results}

\section{A. Influence of the Resolution Bandwidth}

If the power of a single signal has to be characterized, the most obvious choice for the resolution bandwidth is wider than the channel bandwidth. However, when multiple channels are present, the resolution bandwidth should be constrained in order to minimize the contribution of the GSM channel to its neighboring channels. To study the dependence of the measured power level on the resolution bandwidth, the measurement of one GSM signal was simulated 200 times for each resolution bandwidth. The span $\Delta f_{\mathrm{sp}}$ was chosen such that the number of frequency bins per GSM channels $\left(N_{\mathrm{pts}}\right)$ is 10 , or thus that the width of a frequency bin equals $20 \mathrm{kHz}$. The sweep rate was set to $v_{\mathrm{sw}}=200 \mathrm{MHz} / \mathrm{s}$, which means that the resolution filter is not in steady state for resolution bandwidths RBW $<25 \mathrm{kHz}$ [see (6)].

Since there are multiple frequency bins in a GSM channel, and each frequency bin corresponds to a measurement value, an unambiguous method is needed to determine the power present in a GSM channel from the $N_{\text {pts }}$ measurement values in each GSM channel. The method is chosen analogously to the operation of the detector used in the spectrum analyzer:

- For positive peak detection: The measured power is estimated as the maximum of the $N_{\text {pts }}$ measured power values.

- For RMS detection: The measured power of the channel is estimated as the mean RMS power over the $N_{\text {pts }}$ frequency bins.

These measurement values can be determined for each simulated measurement. The mean value of the relative power difference between the power level indicated by the spectrum analyzer and the actual signal power for these 200 samples and its 95\% confidence interval is plotted in Fig. 3. In Fig. 3 this is also shown for 200 traces measured with the spectrum analyzer. From Fig. 3, it can be observed that there is a good agreement between the results from the simulation model and the results from an actual spectrum analyzer measurement.

Fig. 3 shows that the confidence interval of the measured power level narrows for larger resolution bandwidths. Since larger resolution bandwidths correspond to shorter impulse answers, it can be deducted from (7) that the signal sequences determining the measured power level will be shorter. Shorter signal sequences mean that fewer bits determine the measured power level, so the number of possible relevant bit sequences is smaller and, thus, the measured power level will show a smaller variance. When the resolution bandwidth is larger than the signal bandwidth, the number of bits that contribute to

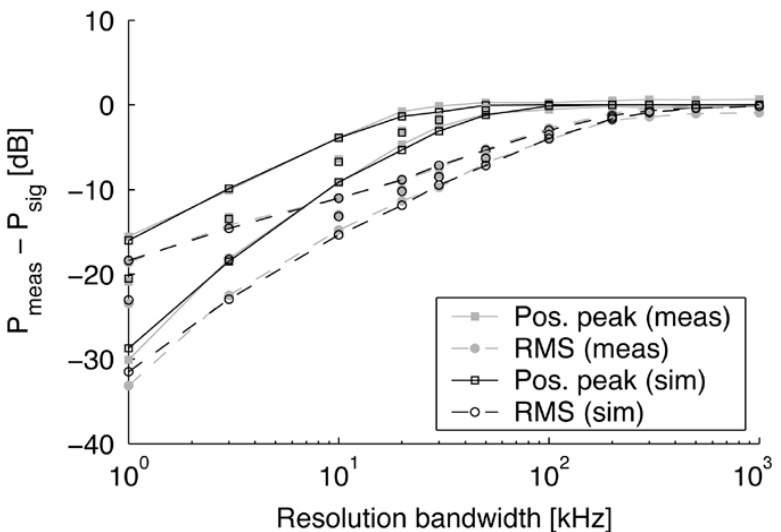

Fig. 3. Influence of the resolution bandwidth on the measured power value. The mean relative difference between the measured power level and the signal power are indicated for the RMS (circle) and positive peak detector (square), together with their $95 \%$ confidence intervals (indicated with a full, resp. dashed line for the positive peak, resp. RMS detector). The results from both the simulation model (black) as well as from the spectrum analyzer measurements (gray) are shown $\left(v_{\mathrm{sw}}=200 \mathrm{MHz} / \mathrm{s}, \Delta f_{\text {bin }}=20 \mathrm{kHz}\right)$.

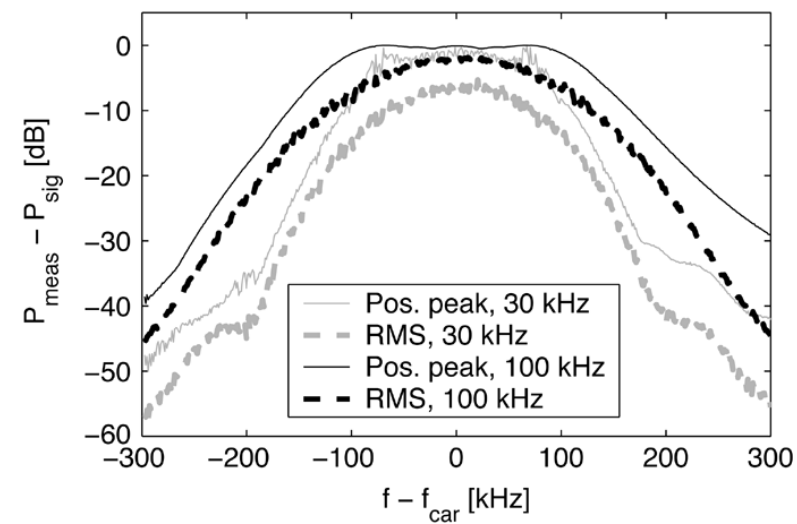

Fig. 4. Influence of the resolution bandwidth on the smoothness of the measured power trace. The positive peak and RMS power traces are plotted for a resolution bandwidth of 30 and $100 \mathrm{kHz}\left(v_{\mathrm{sw}}=1.2 \mathrm{MHz} / \mathrm{s}, \Delta f_{\mathrm{bin}}=\right.$ $1.2 \mathrm{kHz}$ ).

the measured power level is only determined by the GMSK modulation, not by the resolution filter. In Fig. 3, it can also be observed that even for resolution bandwidths wider than the channel bandwidth, there is still a small underestimation of the power level for the RMS detector, since the RMS measured value takes into account the measured values of all the $N_{\text {pts }}$ frequency bins, including the frequency bins not exactly at the carrier frequency. A better estimation of the GSM channel power for wide-resolution bandwidths could be the power measured at the carrier frequency.

A phenomenon analogous to the narrowing of the $95 \%$ confidence interval for increasing resolution bandwidths can be observed when the frequency bin is chosen much smaller than the bandwidth of the GSM channel, i.e., when the number of measurement points per GSM channel $N_{\mathrm{pts}}$ is large. Since for large enough resolution bandwidths (i.e., RBW $>25 \mathrm{kHz}$, for $\left.v_{\mathrm{sw}}=200 \mathrm{MHz} / \mathrm{s}\right)$ the measurement values in different frequency bins are uncorrelated, the measured power trace of a GSM signal will be smoother for wide-resolution filters, as is illustrated in Fig. 4.

In Fig. 4, it can be noticed that at the upper sideband of the GSM channel, the positive peak trace is elevated compared to 
the lower sideband. This effect is due to the guard interval between two successive time slots. During the guard period, the transmitted power of the burst is attenuated by $70 \mathrm{~dB}$, which means that the signal at the input of the spectrum analyzer vanishes. The instantaneous frequency of the signal seen at the input of the resolution filter will lower during the guard period. If the frequency of the local oscillator is below the carrier frequency of the considered signal, this will result in an attenuation of the power level at the output of the resolution filter, while for frequencies higher than the carrier frequency, an elevated power level is seen by the envelope detector. For frequencies in the lower band of the GSM channel, the positive peak detector holds on the power level measured during transmission, while in the upper band, the increased power during the guard interval is retained, resulting in an elevated power on the display for frequencies higher than the carrier frequency. Because the RMS detector averages the power over the whole burst, the detector does not observe the temporary power increase measured during the guard interval. For larger resolution bandwidths (e.g., RBW $>300 \mathrm{kHz}$ ), the time extent of the resolution filter is too short compared to the power variation during the guard interval, such that the signal at the output of the resolution filter follows the power variation of the time slot. In Fig. 4, the attenuation during the guard interval is supposed to be $70 \mathrm{~dB}$. When the measuring antenna is located in a position with strong multiple path fading, the pulse will broaden due to the different times of arrival of each ray, which results in a smaller and less steep attenuation during the guard interval. The alteration of the instantaneous frequency will be less distinct and, thus, the elevation of the power level in the upper sideband will be less pronounced.

When the resolution bandwidth is larger than the channel bandwidth, and the frequency of the local oscillator equals the carrier frequency, the signal leaving the resolution filter will be constant. The RMS detector and the positive peak detector will, thus, both measure the exact power level of the GSM signal. The positive peak detector will approach the correct signal power faster than the RMS detector: Since in GMSK, only the phase of the signal is modulated, the signal power is only determined by the amplitude of the carrier. Moreover, the instantaneous magnitude of the GMSK signal is always limited below the amplitude, and whenever the GMSK signal reaches this upper limit, the positive peak detector will retain this maximum value, and the correct power level will be measured. On the other hand, since the RMS signal depends on the entire signal during the frequency bin, the measured power level will be lower for resolution filters that are narrower than the channel bandwidth because the power at frequencies outside the resolution filter will not contribute to the measured value.

In the absence of adjacent GSM channels, the power of a GSM channel can easily be measured by using a large resolution filter covering the whole GSM channel. On the contrary, the power may also be estimated from a measurement with a resolution filter that is obviously too narrow (e.g., $30 \mathrm{kHz}$ ). Indeed, the relation between the RMS power measured with a narrowresolution filter and the total power of the GSM channel is only determined by the GMSK modulation, and thus, the exact power can be determined from the measured power by adding a correction factor. The correction factor can be derived from Fig. 3 .

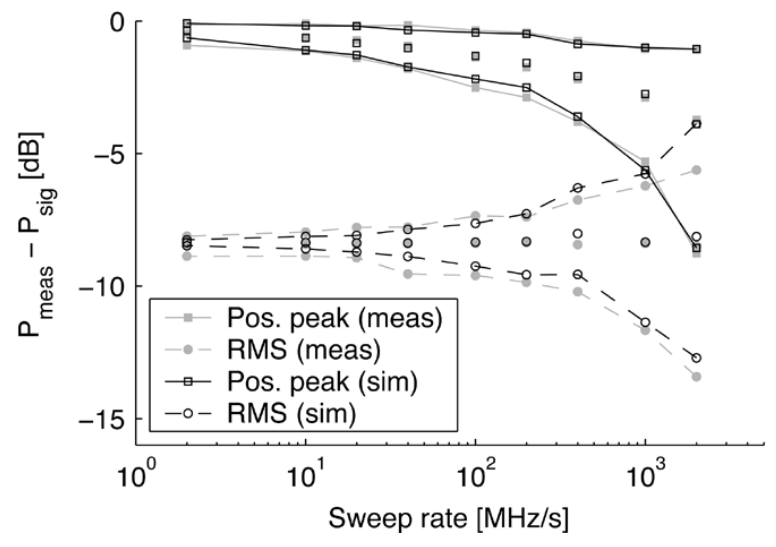

Fig. 5. Influence of the sweep rate on the measured power value. The mean relative power difference between the measured value and the signal power are indicated for the RMS (circle) and positive peak detector (square), together with their $95 \%$ confidence intervals (indicated with a full, resp. dashed line for the positive peak, resp. RMS detector). The results from both the simulation model (black) as from spectrum analyzer measurements (gray) are shown (RBW = $30 \mathrm{kHz}, \Delta f_{\text {bin }}=20 \mathrm{kHz}$ ).

Another option is to measure the GSM channel with the positive peak detector. As is illustrated in Fig. 3, the positive peak detector will measure the correct power level for resolution filters that are much narrower than the GSM channel (for a sweep rate of $v_{\mathrm{sw}}=200 \mathrm{MHz} / \mathrm{s}$, this is possible for resolution bandwidths $\mathrm{RBW} \geq 30 \mathrm{kHz}$ ).

\section{B. Influence of the Sweep Rate}

The variation of the measured power level will decrease when the sweep rate is lowered, as can be seen in Fig. 5. The maximum sweep rate to guarantee a proper response from the resolution filter with RBW $=30 \mathrm{kHz}$ is approximately $300 \mathrm{MHz} / \mathrm{s}$. Fig. 5 again shows a good agreement between the results obtained from the simulation model and the results derived from spectrum analyzer measurements on a GSM channel.

When a lower sweep rate is chosen, the frequency bin will correspond to a longer period in the time domain. More relevant signal sequences will, thus, be considered to determine the measurement value of the frequency bin. Because of the law of large numbers, the variation on the measured RMS value will decrease if a larger number of signal sequences contributes to this measured value. The $95 \%$ confidence interval of the power measured with the positive peak detector will also narrow for lower sweep rates. Since more signal sequences are considered to obtain the positive peak value when the sweeping is slower, it will be more probable that a signal sequence corresponding to the maximum possible peak value will occur. The other way round, the measured RMS value depends on the whole distribution of the measured values, while the positive peak value depends only on the tail distribution. For larger durations of the frequency bin, the distribution of the RMS value will narrow since more signal sequences are contributing to the RMS value, while for the positive peak detector, slower sweeping results in a higher probability that a measured value in the tail distribution occurs. The narrowing of the distribution explains why the mean measured value of the RMS detector remains roughly the same for different sweep times. For the positive peak detector, 
the mean will increase for lower sweep rates, since the probability that a value close to the maximum has appeared increases and, thus, the weight of the distribution of the maximum will shift to the upper limit.

If the power spectrum of one GSM channel is measured by taking very narrow frequency bins (e.g., $\Delta f_{\text {bin }}=2 \mathrm{kHz}$ ), the coarseness of the power trace will also depend on the sweep rate: When the local oscillator sweeps slowly, the power trace will be much smoother.

The situation where multiple sweeps are taken to obtain the maximum value that has occurred during the measuring interval, e.g., when the spectrum analyzer is used in maximum-hold mode, is analogous to a longer sweep time, although the sweep rate remains constant. Because more signal sequences are taken into account to determine the measured positive peak value and the RMS value, the resulting power trace will be smoother.

The choice of the frequency bin width $\Delta f_{\text {bin }}$ will have no influence on the variation of the measurement values if the sweep rate is kept constant. Since the definitions of the measurement values for a frequency channel are in accordance with the detector operation, the usage of more frequency bins per frequency channel will have no influence on the distribution of the measurement values when the sweep rate is kept constant. On the other hand, when more frequency bins are used, the visual distinction between two adjacent channels will be easier.

\section{Effect of the Number of Active Time Slots}

Until now, the GSM channel had been modeled as continuously active. However, the base station can alter its transmitted power depending on the number of active connections. When down link power control is enabled, the power transmitted in a certain time slot will even depend on the reception quality perceived by the corresponding mobile user. Hence, the distribution of the measured power level will depend on the number of active time slots and on the power levels at which they are transmitted. In this paper, only the number of active slots has been examined. The dependence on the number of active slots will be more apparent for the RMS signal, since the RMS detector averages out over time, and thus, over the number of active time slots. Simulations have been carried out for a resolution bandwidth of $30 \mathrm{kHz}$ (Fig. 6) and for a varying number of active time slots.

From Fig. 6, it can be observed that the variation on the measured positive peak and RMS values decreases somewhat when more slots are active: When more slots are being transmitted at maximum power, the probability that a signal sequence corresponding to a maximum level occurs, will become larger, since the number of relevant bit sequences is larger. It is also shown that the positive peak detector has the advantage of always detecting the maximum power that has been emitted during all time slots, while the RMS detector measures the average over active and idle time slots. When the power measurements are used to check compliance with the exposure guidelines, the measurement with the positive peak detector is preferred, since it indicates the highest exposure that has occurred during the measurement time. When not all slots are active, the signal measured with the positive peak detector only undergoes a small attenuation compared to the maximum transmitted power.

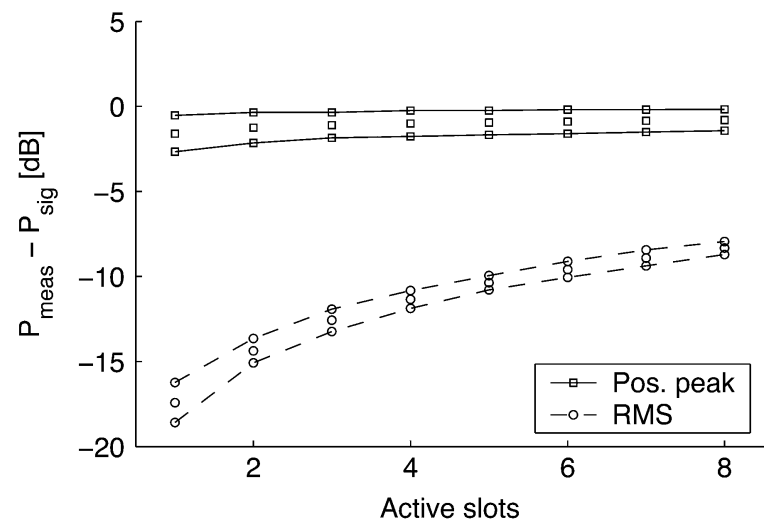

Fig. 6. Influence of the number of active time slots out of eight on the measurement value for the positive peak signal and the RMS signal $(\mathrm{RBW}=30 \mathrm{kHz}$, $\Delta f_{\mathrm{bin}}=20 \mathrm{kHz}$.

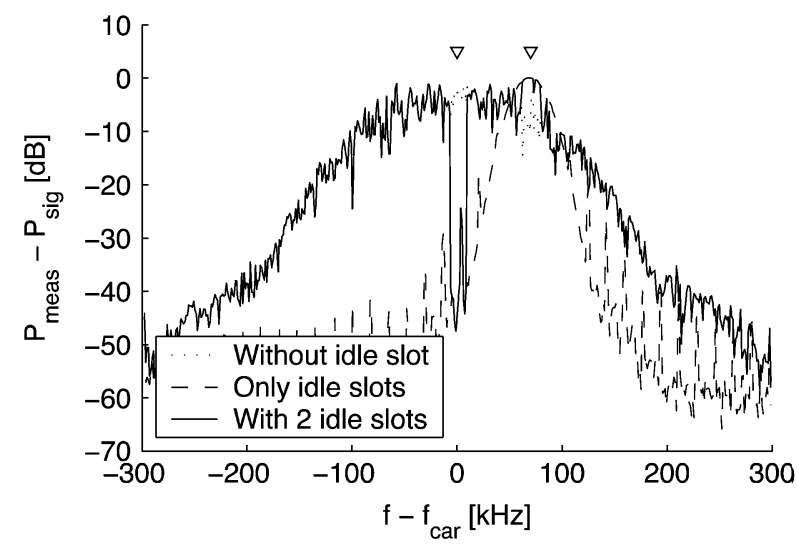

Fig. 7. Influence of the transmission of a constant bit sequence on the measured power trace: Positive peak traces for a series of ordinary time slots (gray, dotted), a series of time slots containing only constant bit sequences (gray, dashed), and for a series of time slots with two time slots where a constant bit sequence was transmitted (indicated by $\nabla$ ). The resolution bandwidth is $30 \mathrm{kHz}$ and the sweep time $\Delta T_{\mathrm{sw}}=20 \mathrm{~ms}\left(v_{\mathrm{sw}}=30 \mathrm{MHz} / \mathrm{s}\right.$, $\Delta f_{\mathrm{bin}}=1.2 \mathrm{kHz}$ ).

\section{Transmission of Constant Bit Sequences}

In normal bursts, the bit sequence that is transmitted by GMSK modulation is a combination of a random bit sequence and a training sequence, resulting in a typical power spectrum given in Fig. 4. However, the base station can also transmit a dummy bit sequence of all the same bits (e.g., in an idle slot on a control channel). There is no training sequence added, since the mobile station does not listen to this idle slot. The GMSK modulator will translate this constant bit sequence in a single tone signal at a frequency of $f_{\text {car }}+70 \mathrm{kHz}$, where $f_{\text {car }}$ is the carrier frequency of the GSM channel. During this particular time slot, the spectrum analyzer will measure this single tone. The measured power level during that time slot will, thus, depend on the frequency of the local oscillator: It will follow the trace of the resolution filter centered at the frequency of the single tone.

This is illustrated in Fig. 7: The measured power spectrum is shown for the situation where only normal bursts are transmitted, for a theoretical sequence of slots where only constant bit sequences are transmitted, and for a series of time slots where in two slots a constant bit sequence was transmitted. As it can be seen in Fig. 7, the power level measured when the local oscillator is not at the single tone frequency, will be much lower com- 


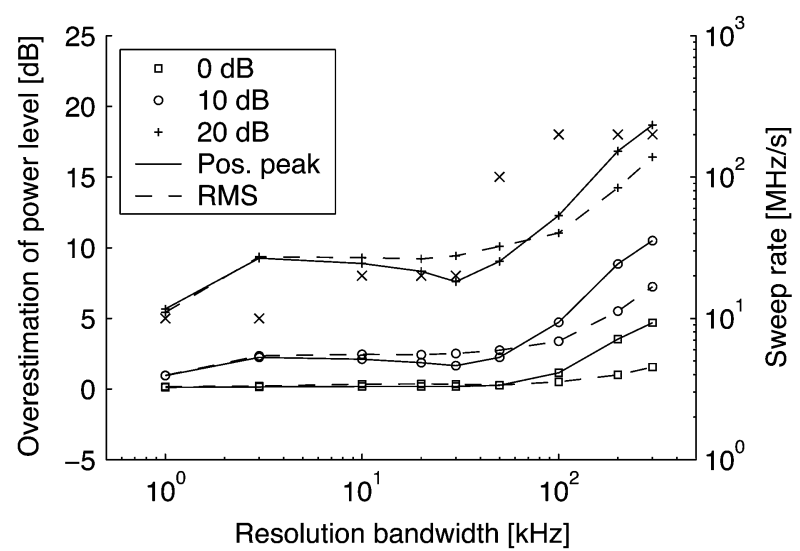

Fig. 8. Influence of the resolution bandwidth on the resolution quality: Overestimation of the power level of the considered channel when an adjacent channel with a power difference of $0 \mathrm{~dB}, 10 \mathrm{~dB}$, and $20 \mathrm{~dB}$ is present. The chosen sweep rate $v_{\mathrm{sw}}$ for each resolution bandwidth is indicated with $\times\left(\Delta f_{\mathrm{bin}}=\right.$ $20 \mathrm{kHz}$ ).

pared to the power level of an ordinary time slot. On the other hand, if the frequency of the local oscillator is at $f_{\text {car }}+70 \mathrm{kHz}$, the measured power level will be the power level of the carrier (irrespective of the used resolution bandwidth since it is a single tone), which will be higher than the power level at that frequency of a normal burst. Both the RMS and the positive peak detector will measure this elevation, provided that the duration of the frequency bin $\Delta f_{\mathrm{bin}} / v_{\mathrm{sw}}$ is shorter than the duration of the slot. Care should be taken when the actual power level of the GSM channel is estimated by correcting the measured power level with a certain factor (e.g., for a resolution bandwidth $\mathrm{RBW}=30 \mathrm{kHz}$ and the RMS detector), since it is possible that there is no need for any correction if the power of the single tone is measured. On the other hand, when the sweep rate is sufficiently slow, the RMS detector will average over several time slots, and the overestimation of the measured power level is negligible.

\section{E. Resolution of Adjacent Channels}

Since measurements to check compliance of base stations with the exposure regulations should be able to distinguish the contributions of different sources, the resolution capability of the spectrum analyzer is of prime importance. The resolution bandwidth is the most determining parameter for the ability of the spectrum analyzer to resolve two adjacent GSM channels. To quantify the resolution quality, a measure has been defined as follows. First, the mean measured power of the considered channel is determined when no neighboring channel is present (averaged over 200 measurement traces). Second, the mean measured power is calculated when a strong adjacent channel is present (with a relative power difference of $0 \mathrm{~dB}$ to $20 \mathrm{~dB}$ ). The measure is then defined as the mean ratio (expressed in decibels) of the power level, when the adjacent channel is present, to the power level of the channel alone. The influence of the resolution bandwidth on the measure for the resolution quality is shown in Fig. 8.

As it can be seen in Fig. 8, the resolution quality of the positive peak detector starts to deteriorate for resolution bandwidths larger than $50 \mathrm{kHz}$, while the resolution quality of the RMS detector remains the same up to $100 \mathrm{kHz}$. It is also shown that the
RMS detector has a better resolution capability than the positive peak detector for resolution filters wider than $100 \mathrm{kHz}$. As expected, when the power difference between the two adjacent channels is higher, the error on the measured power level of the weakest channel is larger (resulting in a worse resolution quality).

\section{SPECTRUM ANALYZER SETtingS}

For the choice of the resolution bandwidth a compromise has to be made between the accuracy of the power measurement and the capability to resolve two adjacent channels. If a resolution filter is used that does not cover the whole GSM channel, the measured power level has to be corrected for the used resolution bandwidth and the used detector mode. It is preferred to use a resolution bandwidth smaller than $50 \mathrm{kHz}$, since this enables to distinguish two adjacent channels with a relative power difference of $10 \mathrm{~dB}$ with an error smaller than $2 \mathrm{~dB}$.

When the compliance of a base station with exposure guidelines has to be demonstrated, it is necessary that the exposure at the considered measurement point will always be below the exposure limits. Therefore, the positive peak detector is preferred since it is able to determine the maximum measured power of a time intermittent signal (e.g., when there is only one time slot active on the frequency channel during the measurement). Moreover, the measured power level for a narrow-resolution filter will be close to the total power of the GSM signal when a positive peak detector is used. The positive peak detector also provides more protection against the overestimation of the power level when a constant bit sequence was transmitted.

The sweep rate should certainly be chosen below the critical value mentioned in (6), but lower sweep rates are even preferred since they result in narrower confidence intervals for the measured values and smoother power traces. If the measured values are obtained during multiple sweeps, there will be less variation on the measured value, provided that the power of the signal remained constant. Moreover, if multiple consecutive sweeps are used, the different traces are less dependent on the instantaneous traffic present on a channel. It is preferred to make $N_{\text {sw }}$ different sweeps in stead of decreasing the sweep rate by a factor $N_{\mathrm{sw}}$, since the measurement value for a single channel will be less dependent on the momentary traffic when several sweeps are taken to obtain a measurement value instead of one slow sweep.

The authors propose to use a resolution filter of $30 \mathrm{kHz}$ at a sweep rate of maximum $20 \mathrm{MHz} / \mathrm{s}$, preferably repeated ten times to narrow the $95 \%$ confidence interval, and to use the positive peak detector to make good exposure assessment measurements around base stations. Lower sweep rates and more consecutive measurements will further narrow the confidence interval.

In Table I, the mean relative power difference between the measured power level and the signal power level, together with the width of the $95 \%$ confidence interval and the resolution quality (i.e., the overestimation of the power level when a $10 \mathrm{~dB}$ stronger adjacent channel is present) are given for the proposed spectrum analyzer settings $(\mathrm{RBW}=30 \mathrm{kHz}$, positive peak detection and $\Delta f_{\text {bin }}=20 \mathrm{kHz}$ ). Three different cases with varying sweep rates $v_{\mathrm{sw}}$ and different number of sweeps $N_{\mathrm{sw}}$ are considered. As expected, the mean power level and the width 
TABLE I

ACCURACY OF PROPOSED SPECTRUM ANALYZER SETtingS

\begin{tabular}{lc|rc|c}
\hline Sweep rate $v_{s w}$ & {$[\mathrm{MHz} / \mathrm{s}]$} & \multicolumn{2}{|c|}{20} & 2 \\
\hline Number of sweeps $N_{s w}$ & & 1 & 10 & 1 \\
\hline \hline Mean power difference & {$[\mathrm{dB}]$} & -0.8 & -0.4 & -0.4 \\
$95 \%$ confidence interval & {$[\mathrm{dB}]$} & 1.1 & 0.6 & 0.6 \\
Resolution quality $(10 \mathrm{~dB})$ & {$[\mathrm{dB}]$} & 1.8 & 1.7 & 1.6 \\
\hline
\end{tabular}

of the $95 \%$ confidence interval are the same for the case where ten sweeps are taken as for the situation where the sweep rate $v_{\mathrm{sw}}$ was chosen ten times lower. It can also be noticed from Table I that the resolution quality is the same for the three different cases.

\section{CONCLUSION}

When frequency selective measurements are executed to determine the worst-case exposure to electromagnetic fields around a base station, the spectrum analyzer settings should be chosen accurately to obtain valid measurement results. Based on a simple simulation model for the spectrum analyzer and for the GSM channel, the optimal choice for these parameters has been made. A resolution bandwidth of $30 \mathrm{kHz}$ has been proposed as a good compromise between resolution and power level accuracy. Although the obtained power level should be corrected for the use of a narrow-resolution filter, the use of the positive peak detector already provides a good estimate of the maximum measured power even when the resolution filter is obviously too narrow. Moreover, the positive peak detector is able to measure the maximum power for time intermittent signals. Since a low sweep rate provides a smaller error on the signal, the sweep rate should be as low as reasonably achievable. On the other hand, the use of multiple sweeps is preferred compared to a slower sweep rate, since the measurement result for a GSM channel is then less dependent on the instantaneous traffic on the GSM channel.

\section{REFERENCES}

[1] "Council recommendation of 12 July 1999 on the limitation of exposure of the general public to electromagnetic fields $(0 \mathrm{~Hz}-300 \mathrm{GHz}), "$ Official J. European Communities, no. L199, pp. 59-70, 1999. European Council.

[2] "Guidelines for limiting exposure to time-varying electric, magnetic, and electromagnetic fields (up to $300 \mathrm{GHz}$ )," Health Physics, International Commission on Non-Ionizing Radiation Protection (ICNIRP), vol. 74, no. 4, pp. 494-594, Apr. 1998.

[3] Electromagnetic Fields $(300 \mathrm{~Hz}$ to $300 \mathrm{GHz})$, ser. Environmental Health Criteria, Geneva, Switzerland: World Health Organization. UNEP/WHO/IRPA.
[4] IEEE Standard for Safety Levels with Respect to Human Exposure to Radio Frequency Electromagnetic Fields, $3 \mathrm{kHz}$ to $300 \mathrm{GHz}$, 1999. IEEE Standard C95.1-1991.

[5] C. Olivier and L. Martens, "A practical method for compliance testing of base stations for mobile communications with exposure limits," in Proc. IEEE Ant. and Prop. Soc./URSI Symp., vol. 2, Boston, MA, Jul. 8-13, 2001, pp. 64-67.

[6] A.-M. Martínez-González, A. Fernández-Pascual, E. de los Reyes, W. Van Loock, C. Gabriel, and D. Sánchez-Hernández, "Practical procedure for verification of compliance of digital mobile radio base stations to limitations of exposure of the general public to electromagnetic fields," in Proc. Microwaves, Ant., Prop. IEE , vol. 149, Aug. 2002, pp. 218-228.

[7] G. Neubauer, H. Haider, and K. Lamedschwandner, "Measurement methods and legal requirements for exposure assessment next to GSM base stations," in Proc. 15th Int. Zurich Symp. Electromagnetic Compatibility, Feb. 18-20, 2003, pp. 143-148.

[8] C. Rauscher, Fundamentals of Spectrum Analysis, München, Germany: Rohde and Schwarz, 2001.

[9] Multiplexing and Multiple Access on the Radio Path. Digital cellular telecommunications system (Phase 2+), ETSI/3GPP Standard TS 05.02

[10] Channel Coding. Digital cellular telecommunications system (Phase 2+) ETSI/3GPP Standard TS 05.03.

[11] Modulation. Digital cellular telecommunications system (Phase 2+), ETSI/3GPP Standard TS 05.04.

[12] Radio Transmission and Reception. Digital cellular telecommunications system (Phase 2+), ETSI/3GPP Standard TS 05.05.

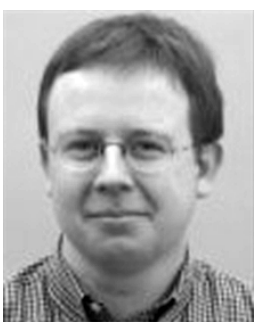

Christof Olivier was born in Izegem, Belgium, on September 24, 1976. He received the M.Sc. degree in electrical engineering from Ghent University, Ghent, Belgium, in 1999, and is currently pursuing the $\mathrm{Ph} . \mathrm{D}$. degree with the Department of Information Technology (INTEC), Ghent University, as a Research Assistant of the Fund for Scientific Research, Flanders, Belgium (F.W.O.-Vlaanderen).

Since August 1999, he has been working on electromagnetic field measurements around base stations for mobile communications related to the health effects of the exposure to electromagnetic radiation. His interests are in electromagnetic field measurements, antennas, and propagation.

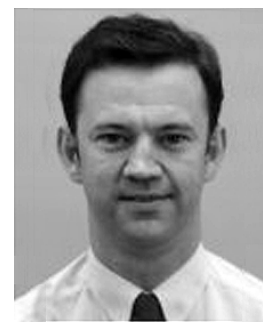

Luc Martens (M'92) was born in Ghent, Belgium, on May 14, 1963. He received the M.Sc. degree in electrical engineering from Ghent University, Belgium, in July 1986.

From September 1986 to December 1990, he was a Research Assistant at the Department of Information Technology (INTEC), Ghent University. During this period, his scientific work was focused on the physical aspects of hyperthermic cancer therapy. His research work dealt with electromagnetic and thermal modeling and with the development of measurement systems for that application. This work led to a Ph.D. degree in December 1990 Since January 1991, he has been a member of the permanent staff of the Interuniversity MicroElectronics Centre (IMEC), Ghent, and is responsible for the research on experimental characterization of the physical layer of telecommunication systems at INTEC. His group also studies topics related to the health effects of wireless communication devices. Since April 1993, he has been a Professor in electrical applications of electromagnetism at Ghent University. 